\title{
Estimating Phenotypic and Genotypic Path Coefficient, an Application on Wheat (Triticum aestivum L.) Genotypes
}

\author{
Sahar A. Farag ${ }^{1}$, S.K.A. Ismail ${ }^{2}$ and Sawsan A. El-Ssadi ${ }^{3 *}$ \\ ${ }^{1}$ Central Laboratory for Dresden and Statistical Analysis Research, of Agriculture, \\ Research Center-Giza-Egypt \\ ${ }^{2}$ Department of Agronomy, Faculty of Agriculture, Fayoum University, Egypt \\ ${ }^{3}$ Department of Agronomy, Faculty of Agriculture, Cairo University, Egypt
}

*Corresponding author

\section{A B S T R A C T}

\section{Keywords}

ANOVA, Path analysis, Genotypic path, Phenotypic path, Wheat

Article Info

Accepted:

24 January 2018

Available Online:

10 February 2018
Two field experiments were carried out at agriculture research stations of Agriculture Research Center (ARC). In 2014/2015 -2015/2016 seasons, the six parental genotypes were planted and the six parents were planted in a field experiment using the randomized complete block design with three replications. With the trials conducted, the correlation coefficients and path analysis were calculated between grain yield and yield components of wheat genotypes. The results revealed that that Gimaza-7, 10 and Misr1 cultivars were the best yielding among the studied cultivars and the Kafr Elhamam location was the top yielding than the other location (Itay-Elbarood). Positive and significant genotypic and phenotypic correlation was found between yield and No. of tillers per plant (NTP), No. of spikelets/ spike (NSS) and No. of spike /plant. Also, results indicated that, based on path analysis, it could be concluded that the most important sources of variation in grain yield. Genotypic and phenotypic coefficient of variation was high for No. of spike per plant and No. of tillers/plant, suggested that these yield components may be a good selection criteria to improve yield of wheat genotypes.

\section{Introduction}

Wheat (Triticum aestivum L.) is one of the most important and strategically over the world. In Egypt, wheat is the main cereal crop used as stable food for urban and rural societies and the major source of straw for animal feeding. However, total wheat consumption has drastically increased due to over. Population growth by about $2.5 \%$ per year. Egypt imports about $45 \%$ of its wheat requirement. This reflects size of the problem and the efforts needed to increase wheat population. Thus, increasing production per unit area appears to be one of the important factors for narrowing the gab between wheat production and consumption.

Improving yield is one of the most important goals for most breeders and geneticists working with quantitative traits. Grain yield is a highly polygenic quantitative character that is greatly affected by environmental fluctuations. Grain yield in wheat, as in other 
crops, is a complex character, which resulted as the sum-total of the contributions made by its individual components. Grafius (1959) has even doubted the individuality of grain yield. Yield and some of its components, such as the number of spikes per plant, cannot be wholly reliably used as criteria for selection because of their low heritability and wide fluctuations as a result of their interaction with the environment. Yield, as a function of various components, is a complex character. It was suggested that yield is a function of various components, where it depends on the number of spikes per unit area, the number of wheat grains per spike and the average grain weight. The grain yield and yield components of wheat are affected very much by the genotype and the environment. Therefore, as new cultivars are being produced by breeding, the relationships between grain yield and its components should be studied by the breeders. To increase the yield, studding the direct and indirect effects of yield components on yield provides the basis for its successful breeding programme and hence the problem of yield increase can be more effectively tackled through exploitation the performance of yield components and selection for closely related characters (Mehmet and Telat, 2006). Based on path analysis, it could be determined the most important sources of variation in grain yield. Significant genotypic and phenotypic variances, differentiated the yield components which may be used as good selection criteria to improve grain yield of wheat genotypes. The aim of this study was to determine the correlations and path analysis of yield and yield components in bread wheat and evaluate their suitabilities in a breeding program.

\section{Materials and Methods}

Two variance-covariance matrices necessary for calculating genotypic and phenotypic correlation coefficients as well as for evaluating the path coefficients technique, were obtained from the mean squares and mean cross products of genotypes together with error for different characters measured in a replicated experiment.

Analysis of variance for each character and analysis of covariance for all pairs of the studied characters were constructed separately. The expectations of mean squares and mean cross products are given in Table 1.

\section{Mean squares were used to estimate}

$\sigma_{\mathrm{g}}^{2}=(\mathrm{MSS}-\mathrm{MSE}) / \mathrm{r}$

$\sigma_{\mathrm{ph}}{ }^{2}=\sigma_{\mathrm{e}}{ }^{2}+{\sigma_{\mathrm{g}}}^{2}$, where broad-sense heritability $\left(\mathrm{h}^{2}\right)$ was estimated as follows:

$\mathrm{h}^{2}=\left(\sigma_{\mathrm{g}}{ }^{2} / \sigma_{\mathrm{ph}}{ }^{2}\right) \times 100$, and the phenotypic, genotypic and environmental coefficients of variation are computed as follows:

$\mathrm{GCV}=100 x \sqrt{\sigma_{g}^{2}} / \overline{-}$

$\mathrm{ECV}=100 x \sqrt{\sigma_{e}^{2}} / X^{-}$

The same mean products were used to estimate

$\sigma_{\mathrm{gij}}=(\mathrm{MPS}-\mathrm{MPE}) / \mathrm{r}$

$\sigma_{\text {phij }}=\sigma_{\text {eij }}+\sigma_{\text {gij }}$, where phenotypic, genotypic and environmental correlation coefficients are computed as follows:

$\mathrm{r}_{\mathrm{ph}}=\sigma_{p h i j} / \sqrt{\sigma_{p h i}^{2} x \sigma_{p h j}^{2}}$

$\mathbf{r}_{\mathrm{g}}=\sigma_{g i j} / \sqrt{\sigma_{g i}^{2} x \sigma_{g j}^{2}}$

$\mathrm{r}_{\mathrm{e}}=\sigma_{e i j} / \sqrt{\sigma_{e i}^{2} x \sigma_{e j}^{2}}$.

Expected genetic advance: Expected genetic 
advance from direct selection for all studied traits was calculated according to (Sing and Chaudhary 1999) as follows:

$\square G \%=100 \mathrm{X} \mathrm{kX} \mathrm{h}^{2} \mathrm{X} \sigma_{\mathrm{ph}} / \overline{\bar{x}}$,

Where, $\bar{X}$ : general mean and $\mathrm{k}$ is selection differential ( $k=1.76$ for $10 \%$ selection).

Calculation of all possible simple correlation coefficients among various characters included in the study, which is equal to $n$ (n$1) / 2$, where $n$ is the number of characters as in Table 2.

The path analysis is carried out according to (Wright, 1921, Dewey and Lu, 1959 and Singh and Narayanan 2000), let $\mathrm{n}$ independent variables be significantly correlated with dependent variable $\mathrm{Y}$ then the correlation matrices representing correlation coefficients (phenotypic, genotypic and environmental) are given in Table 2.

The correlation coefficient between $i^{\text {th }}$ independent variables and dependent variable $\mathrm{Y}$ is linearly related with the correlation coefficients of $\mathrm{i}^{\text {th }}$ independent variable with remaining independent variables.

The relation is denoted as follows:

$r_{i y}=P_{1 y} r_{i 1}+P_{2 y} r_{i 2}+\ldots+P_{n y} r_{n y}$. For $i=1, \ldots$, n.

Where $\mathrm{P}_{1 \mathrm{y}}, \mathrm{P}_{2 \mathrm{y}}, \ldots, \mathrm{P}_{\mathrm{ny}}$ are the coefficients in the linear relation and are known as path coefficients. $P_{i y}$ is called the direct effect of $i^{\text {th }}$ independent characters $\left(\mathrm{Ch}_{\mathrm{i}}\right)$ and dependent variable $\mathrm{Y} . \mathrm{P}_{1 \mathrm{y}} \mathrm{r}_{\mathrm{i} 1}, \mathrm{P}_{2 \mathrm{y}} \mathrm{r}_{\mathrm{i} 2}, \ldots, \mathrm{P}_{\mathrm{ny}} \mathrm{r}_{\text {in }}$ are called the indirect effects of $\mathrm{Ch}_{\mathrm{i}}$ on $\mathrm{Y}$. Therefore the simple correlation coefficient (Total effect) between $\mathrm{Ch}_{\mathrm{i}}$ and $\mathrm{Y}$ is the sum of direct and indirect effects of $\mathrm{Ch}_{\mathrm{i}}$ on $\mathrm{Y}$. The linear relations are represented by matrix notation as: $\mathrm{nxn} \quad \mathrm{nx} 1 \quad \mathrm{n} \times 1$

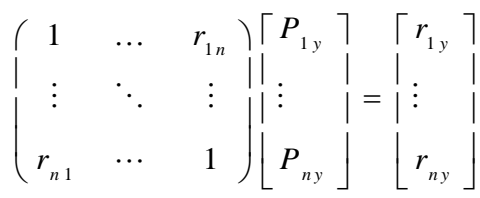

Hence

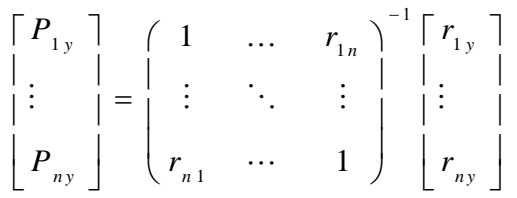

Therefore, the path coefficients are obtained and hence the direct and indirect effects can be obtained. Further, the residual effect is obtained as follows:

$P_{\text {Ry }}=\left(1-\left(P_{1 y} r_{i 1}+P_{2 y} r_{i 2}+\ldots+P_{n y} r_{n y}\right)\right)^{1 / 2}$.

Two field experiments were conducted at agriculture research stations of Agriculture Research Center (ARC) in Egypt to investigate the effect of leaf rust in yield verities, during the two successive growing seasons of 2014/2015 -2015/2016 in two locations (Itay-Elbarood and Kafr Elhamam). Six Egyptian wheat cultivars namely: Gemmeiza7 and Sids-1 (susceptible), Sakha93 (partial resistant), Sakha-94 (resistance), Gemeiza10 and Misr-1 (moderately resistant) were used.

The commercial names, pedigree and origin are presented in Table 3. The cultivars were planted in a field experiment using the randomized complete block design (RCBD) with three replication. Seed of each entry were spaced $10 \mathrm{~cm}$ about in one row $3 \mathrm{~m}$ long and $20 \mathrm{~cm}$ between rows. The cultivars were planted in15th November in both seasons. All agricultural practices were done as usual in wheat fields. All plots were surrounded by a spreader area equal one meter width planted with the highly susceptible. 
Data were recorded on random samples of 10 guarded plants from each row, for PH: Plant height, NTP: No. tillers/plant, FL: Flag Leaf area, NSP: Number of spike/plant, SL: Spike length, NSS: No. spikelets /spike, $1000 \mathrm{GW}$ : weight 1000 grains, Y: Grain yield/ard/fed. Direct and indirect effects of traits were evaluated by correlation and path coefficients. Correlation and path coefficients were calculated by using PATHC Statistical Computer programmed (Atia, 2007).

\section{Results and Discussion}

\section{Analysis of variance and mean performance}

In this experiment, grain yield, plant height, number of tillers/plant, flag leaf area, number of spike/plant, spike length, number of spikelets/spik, and 1000-- grain weight were studied.

The mean square effect of all studied traits was highly significant for all traits in both locations. It is indicates a substantially additive behavior for these traits of variability (Table 4).

The comparison of varieties under two locations showed that all of traits were high level at Kafr Elhamam than Itay-Elbarood (Table 5).

The effect of cultivar on grain yield was significant at 5\% level of significance in both locations (Table 5). This may be due to inherent differences between the cultivars in the yield components like the number of tillers per plant, number of spikelets per spike and 1000 grain weight.

Similar results were reported by Abdel Mohsen et al., 2012. Cultivars Gemmeiza-7 and Gemmeiza-10 produced the highest grain yield (21.29 $\mathrm{ard} / \mathrm{Fed}$. and $20.68 \mathrm{ard} / \mathrm{Fed}$, in Kafr Elhamam and Itay-Elbarood, respectively). The results in table 5 also showed that the cultivars Sids1 and Sakha94 produced the lowest (19.6 ard/fed in ItayElbarood and 18.77 in Kafr Elhamam, respectively) comparing with all evaluated genotypes (Abd El-Kreem and Ahamed 2013).

\section{Genetic parameters}

Genotypic (GCV) and phenotypic (PCV) coefficient of variability, broad sense heritability $\left(\mathrm{h}^{2}\right)$ and genetic advance (EGA) expressed as percent of mean for studied traits, evaluated in six varieties are presented in table 6. High estimates of GCV were obtained by number of spike/plant (20.48 and $17.44 \%$ ) followed by No. of tillers/ plant (19.65 and 9.49\%) in Itay Elbarood and Kafr Elhamam locations, respectively. While moderate values of GCV were observed with plant height and 100-grain weight in both locations.

On the other hand, the values of phenotypic coefficient of variation PCV were slightly higher than their corresponding values of GCV for all traits which reflect somewhat environmental influence on the expression of characters in the two locations. These results indicated that the selection would be effective to improve these traits among the tested genotypes.

The heritability values ranged from $99 \%$ for plant height in both locations and $48 \%$ for No. of spikelets /spik in Itay Elbarood and 23\% flag leaf area length in Kafr Elhamam location, while the values of GA\% ranged between 38.81 for No. of spike /plant and $15.91 \%$ for grain yield in Itay Elbarood and ranged from 34.401 for No. of spike/plant to $8.597 \%$ for flag area in Kafr Elhamam location. Similar results were reported by Kashif and Khaliq (2004), Aycicek and Yildirim (2006), Atia et al., (2007), Ali et al., (2008), Ashmawy et al., (2010), Khan and Dar (2010) and Baloch et al., (2013). 
Table.1 Mean squares (MS), and mean products (MP) from variance-covariance analysis of RCBD

\begin{tabular}{|l|c|c|c|c|}
\hline \multirow{2}{*}{ Source } & \multicolumn{2}{|c|}{ Analysis of Variance } & \multicolumn{2}{c|}{ Analysis of Covariance } \\
\hline Replicates & M.S. & $\begin{array}{c}\text { Expectation } \\
\text { of M.S. }\end{array}$ & M.C.P. & $\begin{array}{c}\text { Expectation } \\
\text { of M.C.P. }\end{array}$ \\
\hline Treatments & $\mathrm{Mr}_{\mathrm{u}}$ & - & $\mathrm{Mr}_{12}$ & - \\
\hline Error & $\mathrm{Mt}_{\mathrm{n}}$ & $\sigma_{\mathrm{e}}{ }^{2}+\mathrm{r}_{\mathrm{g}}{ }^{2}$ & $\mathrm{Mt}_{12}$ & $\sigma_{\mathrm{eij}}+\mathrm{r} \sigma_{\mathrm{gij}}$ \\
\hline
\end{tabular}

*r: number of replications.

Table.2 The all possible simple correlation coefficients (correlation matrix) for phenotypic, genotypic and environmental

\begin{tabular}{|c|c|c|c|c|}
\hline \multicolumn{5}{|c|}{ Phenotypic correlations } \\
\hline & $\mathrm{Y}$ & $\mathrm{Ch}_{1}$ & ... & $\mathrm{Ch}_{\mathrm{n}}$ \\
\hline $\mathbf{Y}$ & 1 & & & \\
\hline $\mathrm{Ch}_{1}$ &.$r_{\mathrm{ph} 1 \mathrm{y}}$ & 1 & & \\
\hline$\ldots$ & $\ldots$ & ... & ... & $\ldots$ \\
\hline $\mathrm{Ch}_{\mathrm{n}}$ &.$r_{\text {phny }}$ &.$r_{\mathrm{ph} 1 \mathrm{n}}$ & ... &. $\mathbf{r}_{\mathrm{phnn}}$ \\
\hline \multicolumn{5}{|c|}{ Genotypic correlations } \\
\hline & $\mathrm{Y}$ & $\mathrm{Ch}_{1}$ & ... & $\mathrm{Ch}_{\mathrm{n}}$ \\
\hline $\mathbf{Y}$ & 1 & & & \\
\hline $\mathrm{Ch}_{1}$ &. $\mathrm{r}_{\mathrm{g} 1 \mathrm{y}}$ & 1 & & \\
\hline$\ldots$ & $\ldots$ & ... & ... & $\ldots$ \\
\hline $\mathrm{Ch}_{\mathrm{n}}$ &.$r_{\text {gny }}$ &.$r_{\mathrm{g} 1 \mathrm{n}}$ & ... &. $\mathbf{r}_{\mathrm{gnn}}$ \\
\hline \multicolumn{5}{|c|}{ Environment correlations } \\
\hline & $\mathrm{Y}$ & $\mathrm{Ch}_{1}$ & $\cdots$ & $\mathrm{Ch}_{\mathrm{n}}$ \\
\hline $\mathbf{Y}$ & 1 & & & \\
\hline $\mathrm{Ch}_{1}$ &. $\mathrm{r}_{\mathrm{e} 1 \mathrm{y}}$ & 1 & & \\
\hline$\ldots$ & ... & $\cdots$ & $\cdots$ & ... \\
\hline $\mathrm{Ch}_{\mathrm{n}}$ &. $\mathbf{r}_{\text {eny }}$ & $\mathbf{r}_{\mathrm{eln}}$ & $\ldots$ &. $\mathbf{r}_{\mathrm{enn}}$ \\
\hline
\end{tabular}

The matrices in Table (2) are symmetric i.e., $r_{i y}=r_{y i}, i=1, \ldots, n$.

Table.3 The commercial names and pedigree of the varieties

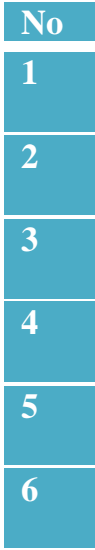

\begin{tabular}{l|l|}
\hline \multicolumn{1}{|c|}{ Pedigree } & origin \\
\hline $\begin{array}{l}\text { CMH74.630/5X//SERI82/3/AGENT } \\
\text { CGM 4611-2GM-3GM-1GM-0GM }\end{array}$ & Egypt \\
\hline $\begin{array}{l}\text { HD2173/PAVON"S"//1158.57/MAYA 74 "S" } \\
\text { SD46-4SD-2SD-1SD-0SD }\end{array}$ & Egypt \\
$\begin{array}{l}\text { SAKHA 92/TR 810328 } \\
\text { S8871-1S-2S-1S-0S }\end{array}$ & Egypt \\
$\begin{array}{l}\text { OPATA/RAYON/3/JUP/BJY//URES } \\
\text { CMBW90Y3180-0EGY }\end{array}$ & Egypt \\
$\begin{array}{l}\text { Ald " S" / Huac// CMH47A.30/SX } \\
\text { CGM4583-5GM-1GM-0GM }\end{array}$ & Egypt \\
$\begin{array}{l}\text { OASIS/SKAUZ//4*BCN/3/2*PASTOR } \\
\text { CMSS00Y01881T-050M-030Y-030M-030WGY-33M-0Y-0S }\end{array}$ & Egypt \\
\hline
\end{tabular}


Table.5 Mean values of grain yield and its related characters for 6 wheat varieties evaluated in adequate and stress locations

\begin{tabular}{|c|c|c|c|c|c|c|c|c|c|c|c|c|c|c|c|c|c|}
\hline \multirow{2}{*}{$\stackrel{\ominus}{Z}$} & \multirow{2}{*}{ 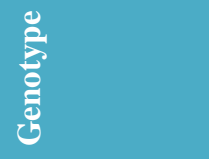 } & \multicolumn{2}{|c|}{$\begin{array}{l}\text { Plant height } \\
(\mathbf{c m})\end{array}$} & \multicolumn{2}{|c|}{$\begin{array}{l}\text { No. of } \\
\text { tillers/plant }\end{array}$} & \multicolumn{2}{|c|}{ Flag Leaf area } & \multicolumn{2}{|c|}{$\begin{array}{l}\text { No. of spike } \\
\text { /plant }\end{array}$} & \multicolumn{2}{|c|}{$\begin{array}{l}\text { Spike length } \\
(\mathrm{cm})\end{array}$} & \multicolumn{2}{|c|}{ No. of kinder } & \multicolumn{2}{|c|}{$\begin{array}{l}\text { Weight of } 1000 \\
\text { grain }(\mathrm{g})\end{array}$} & \multicolumn{2}{|c|}{$\begin{array}{l}\text { Grain Yield /fed } \\
\text { (ard) }\end{array}$} \\
\hline & & $\begin{array}{l}\text { Itay- } \\
\text { Ellbarood }\end{array}$ & $\begin{array}{c}\text { Kafi } \\
\text { Elhamam }\end{array}$ & $\begin{array}{l}\text { Itay- } \\
\text { Ellbarood }\end{array}$ & $\begin{array}{c}\text { Kafi } \\
\text { Elhamam }\end{array}$ & $\begin{array}{l}\text { Itay- } \\
\text { Ellbarood }\end{array}$ & $\begin{array}{c}\text { Kafi } \\
\text { Elhamam }\end{array}$ & $\begin{array}{l}\text { Itay- } \\
\text { Ellbarood }\end{array}$ & $\begin{array}{c}\text { Kafi } \\
\text { Elhamam }\end{array}$ & $\begin{array}{l}\text { Itay- } \\
\text { Elbarood }\end{array}$ & $\begin{array}{c}\text { Kafi } \\
\text { Elhamam }\end{array}$ & $\begin{array}{l}\text { Itay- } \\
\text { Ellbarood }\end{array}$ & $\begin{array}{c}\text { Kafi } \\
\text { Elhamam }\end{array}$ & $\begin{array}{l}\text { Itay- } \\
\text { Elbarood }\end{array}$ & $\begin{array}{c}\text { Kafi } \\
\text { Elhamam }\end{array}$ & $\begin{array}{c}\text { Itay- } \\
\text { Ellbarood }\end{array}$ & $\begin{array}{c}\text { Kafi } \\
\text { Elhamam }\end{array}$ \\
\hline 1 & $\begin{array}{l}\text { GEMMEIZA- } \\
7\end{array}$ & 115.43 & 123.03 & 5.95 & 5.61 & 23.61 & 32.41 & 11.14 & 14.28 & 13.04 & 19.28 & 11.73 & 15.82 & 39.03 & 38.16 & 20.14 & 21.29 \\
\hline 2 & SIDS 1 & 119.18 & 125.23 & 5.18 & 5.67 & 28.03 & 30.32 & 8.05 & 12.04 & 10.15 & 17.22 & 8.48 & 14.37 & 35.15 & 34.05 & 19.60 & 19.89 \\
\hline 3 & SAKHA 93 & 122.35 & 125.69 & 5.12 & 5.70 & 30.11 & 36.59 & 11.08 & 12.85 & 13.46 & 15.27 & 11.85 & 14.18 & 38.60 & 38.92 & 19.61 & 20.98 \\
\hline 4 & SAKHA 94 & 126.50 & 126.43 & 5.30 & 5.75 & 30.62 & 37.90 & 8.40 & 11.69 & 11.82 & 12.58 & 11.25 & 10.90 & 38.99 & 35.93 & 18.77 & 20.99 \\
\hline 5 & $\begin{array}{l}\text { GEMMIEZA- } \\
10\end{array}$ & 120.46 & 124.99 & 3.89 & 5.31 & 30.44 & 36.74 & 9.17 & 14.50 & 16.35 & 16.47 & 13.16 & 15.05 & 37.59 & 40.55 & 20.68 & 20.86 \\
\hline 6 & Misr-1 & 121.85 & 124.76 & 3.99 & 4.89 & 33.73 & 37.70 & 8.10 & 12.42 & 13.55 & 16.42 & 12.67 & 13.58 & 34.89 & 33.80 & 20.41 & 20.27 \\
\hline & LSD & 2.782 & 2.057 & 1.176 & 0.843 & 6.041 & 9.291 & 1.36 & 1.135 & 1.97 & 4.076 & 2.55 & 1.489 & 2.87 & 2.358 & 1.07 & 1.026 \\
\hline
\end{tabular}

Table.6 Genetic parameters of grain yield and its related characters computed from 6 wheat varieties evaluated in two locations

\begin{tabular}{|c|c|c|c|c|c|c|c|c|c|c|c|c|}
\hline \multirow{3}{*}{ Character } & \multicolumn{12}{|c|}{ Genetic parameters } \\
\hline & \multicolumn{2}{|c|}{ Grand mean } & \multicolumn{2}{|c|}{ GCV } & \multicolumn{2}{|c|}{ PhCV } & \multicolumn{2}{|c|}{$h^{2}$} & \multicolumn{2}{|c|}{$\begin{array}{l}\text { EGA (\% mean) at } \\
0.10\end{array}$} & \multicolumn{2}{|c|}{$\begin{array}{l}\text { EGA ( } \% \text { mean }) \text { at } \\
0.5\end{array}$} \\
\hline & IE. & KE. & IE. & KE. & IE. & KE. & IE. & KE. & IE.. & KE. & IE. & KE. \\
\hline Plant height & 120.96 & 125.02 & 13.08 & 13.70 & 13.16 & 13.74 & 0.99 & 0.99 & 22.90 & 24.05 & 26.80 & 28.148 \\
\hline Number of tillers/plant & 4.90 & 5.49 & 19.65 & 9.49 & 24.36 & 13.23 & 0.65 & 0.51 & 27.89 & 11.97 & 32.65 & 14.014 \\
\hline Flag Leaf area & 29.42 & 35.28 & 11.55 & 8.68 & 16.89 & 18.04 & 0.47 & 0.23 & 13.89 & 7.35 & 16.26 & 8.597 \\
\hline Number of spike/plant & 9.32 & 12.96 & 20.48 & 17.44 & 22.26 & 18.22 & 0.85 & 0.92 & 33.16 & 29.39 & 38.81 & 34.401 \\
\hline Spike length & 13.06 & 16.21 & 16.29 & 13.66 & 18.64 & 20.36 & 0.76 & 0.45 & 25.06 & 16.13 & 29.33 & 18.878 \\
\hline Number of spikelets/spike & 11.52 & 13.98 & 12.9 & 11.01 & 18.53 & 12.73 & 0.48 & 0.75 & 15.81 & 16.76 & 18.51 & 19.618 \\
\hline Weight of 1000 grains & 37.37 & 36.90 & 9.11 & 10.01 & 10.21 & 10.72 & 0.80 & 0.87 & 14.29 & 16.46 & 16.73 & 19.261 \\
\hline Grain yield/ ard/fed & 19.87 & 20.71 & 8.29 & 10.65 & 8.89 & 11.06 & 0.87 & 0.93 & 13.59 & 18.05 & 15.91 & 21.129 \\
\hline
\end{tabular}


Table.4 Mean squares for grain yield and other agronomic characters

\begin{tabular}{|c|c|c|c|}
\hline \multirow[t]{2}{*}{ Loc. } & \multirow[t]{2}{*}{ Characters } & \multicolumn{2}{|c|}{ Comb. } \\
\hline & & MSS & MSE \\
\hline \multirow[t]{8}{*}{ Itay-Elbarood } & Plant height & $754.11 * *$ & 2.79 \\
\hline & Number of tillers/plant & $3.28 * *$ & 0.50 \\
\hline & Flag Leaf area & $47.78 * *$ & 13.16 \\
\hline & Number of spike/plant & $11.6 * *$ & 0.66 \\
\hline & Spike length & $14.99 * *$ & 1.41 \\
\hline & Number of spikelets/spike & $8.98 * *$ & 2.35 \\
\hline & Weight of 1000 grains & $37.72 * *$ & 2.98 \\
\hline & Grain yield/ ard/fed & $8.54 * *$ & 0.41 \\
\hline \multirow[t]{8}{*}{ Kafr Elhamam } & Plant height & $881.49 * *$ & 1.53 \\
\hline & Number of tillers/plant & $1.06 * *$ & 0.26 \\
\hline & Flag Leaf area & 59.0 N.S & 31.11 \\
\hline & Number of spike/plant & $15.8 * *$ & 0.46 \\
\hline & Spike length & $20.69 * *$ & 5.99 \\
\hline & Number of spikelets/spike & $7.91 * *$ & 0.80 \\
\hline & Weight of 1000 grains & $42.96 * *$ & 2.00 \\
\hline & Grain yield/ ard/fed & $14.98 * *$ & 0.38 \\
\hline
\end{tabular}

Table.7 Genotypic (above diagonal) and phenotypic (below diagonal) correlation coefficients among grain yield/plant and its related characters computed from 6

wheat varieties evaluated in two locations

\begin{tabular}{|c|c|c|c|c|c|c|c|c|}
\hline Character & PH & NTP & FL & NSP & SL & NSS & 1000 GW & $\mathbf{Y}$ \\
\hline \multicolumn{9}{|c|}{ Itay-Elbarood } \\
\hline PH & 1 & 0.066 & 0.019 & 0.033 & 0.030 & 0.043 & 0.019 & 0.038 \\
\hline NTP & 0.046 & 1 & 0.306 & $0.544 * *$ & -0.080 & -0.328 & 0.305 & $0.433 * *$ \\
\hline FL & 0.053 & 0.210 & 1 & 0.154 & -0.006 & 0.198 & 0.087 & 0.179 \\
\hline NSP & 0.026 & 0.527 & 0.134 & 1 & 0.246 & 0.273 & 0.154 & 0.318 \\
\hline SL & 0.035 & -0.102 & 0.096 & 0.293 & 1 & 0.316 & 0.138 & 0.285 \\
\hline NSS & 0.013 & -0.219 & 0.012 & 0.292 & 0.271 & 1 & 0.198 & $0.407 *$ \\
\hline $1000 \mathrm{GW}$ & 0.010 & 0.305 & -0.015 & 0.184 & 0.137 & 0.187 & 1 & 0.179 \\
\hline $\mathbf{Y}$ & 0.028 & $0.365^{*}$ & 0.123 & 0.251 & 0.210 & 0.217 & 0.183 & 1 \\
\hline \multicolumn{9}{|c|}{ Kafr Elhamam } \\
\hline $\mathbf{P H}$ & 1 & 0.112 & 0.019 & 0.026 & 0.026 & 0.038 & 0.016 & 0.026 \\
\hline NTP & 0.096 & 1 & $0.627 * *$ & $0.708 * *$ & $0.350 *$ & -0.168 & 0.324 & $0.871 * *$ \\
\hline$\overline{F L}$ & 0.022 & $0.514 * *$ & 1 & 0.145 & 0.148 & 0.212 & -0.033 & 0.148 \\
\hline NSP & 0.025 & $0.575^{* *}$ & 0.175 & 1 & 0.200 & 0.287 & 0.120 & 0.201 \\
\hline SL & 0.033 & 0.282 & 0.113 & 0.189 & 1 & 0.293 & 0.005 & 0.205 \\
\hline NSS & 0.026 & -0.155 & -0.139 & 0.226 & 0.203 & 1 & 0.176 & -0.079 \\
\hline 1000 GW & 0.017 & $0.351^{*}$ & -0.019 & 0.115 & 0.103 & 0.138 & 1 & 0.123 \\
\hline $\mathbf{Y}$ & 0.029 & $0.616^{* * *}$ & 0.076 & 0.213 & 0.131 & -0.071 & 0.109 & 1 \\
\hline
\end{tabular}

Abbreviations: PH: Plant height, NTP: No. tillers/plant, FL: Flag Leaf area, NSP: Number of spike/plant, SL: Spike length, NSS: No. spikelets/spike, $1000 \mathrm{GW}$ : weight 1000 grains, Y:Grain yield/ard/fed 
Table.8 The direct and indirect effects of six predictor characters on grain yield/plant at genotypic and phenotypic levels computed from 6 wheat varieties evaluated in two locations

\begin{tabular}{|c|c|c|c|c|c|c|c|c|}
\hline \multirow[t]{2}{*}{ Character } & \multirow[t]{2}{*}{ Level } & \multicolumn{7}{|c|}{ Indirect effects } \\
\hline & & PH & NTP & FL & NSP & SL & NSS & $1000 \mathrm{GW}$ \\
\hline \multicolumn{9}{|c|}{ Itay-Elbarood } \\
\hline \multirow[t]{2}{*}{$\overline{\mathbf{P H}}$} & G & -0.0771 & 0.0966 & -0.0065 & -0.0248 & 0.0082 & 0.0492 & -0.0073 \\
\hline & $\mathbf{P h}$ & -0.0065 & 0.0286 & -0.0002 & -0.0065 & 0.0088 & 0.0047 & -0.0007 \\
\hline \multirow{2}{*}{ NTP } & G & -0.0051 & 1.4729 & -0.1062 & -0.4081 & -0.0222 & -0.3794 & -0.1192 \\
\hline & $\mathbf{P h}$ & -0.0003 & 0.6233 & -0.0008 & -0.1303 & -0.0260 & -0.0807 & -0.0198 \\
\hline \multirow[t]{2}{*}{$\overline{F L}$} & G & -0.0014 & 0.4500 & -0.3477 & -0.1157 & -0.0017 & 0.2291 & -0.0338 \\
\hline & $\mathbf{P h}$ & -0.0004 & 0.1306 & -0.0038 & -0.0331 & 0.0244 & 0.0046 & 0.0010 \\
\hline \multirow[t]{2}{*}{ NSP } & G & -0.0026 & 0.8007 & -0.0536 & -0.7507 & 0.0681 & 0.3163 & -0.0601 \\
\hline & $\mathbf{P h}$ & -0.0002 & 0.3282 & -0.0005 & -0.2475 & 0.0746 & 0.1078 & -0.0119 \\
\hline \multirow[t]{2}{*}{$\overline{\text { SL }}$} & G & -0.0023 & -0.1181 & 0.0021 & -0.1848 & 0.2767 & 0.3657 & -0.0540 \\
\hline & $\mathbf{P h}$ & -0.0002 & -0.0635 & -0.0004 & -0.0724 & 0.2549 & 0.1001 & -0.0089 \\
\hline \multirow[t]{2}{*}{ NSS } & G & -0.0033 & -0.4829 & -0.0688 & -0.2052 & 0.0874 & 1.1573 & -0.0772 \\
\hline & $\mathbf{P h}$ & -0.0001 & -0.1364 & -0.0001 & -0.0723 & 0.0691 & 0.3690 & -0.0121 \\
\hline \multirow{2}{*}{$1000 \mathrm{GW}$} & G & -0.0014 & 0.4493 & -0.0301 & -0.1156 & 0.0382 & 0.2288 & -0.3907 \\
\hline & $\mathbf{P h}$ & -0.0001 & 0.1898 & 0.0001 & -0.0455 & 0.0349 & 0.0690 & -0.0649 \\
\hline \multicolumn{9}{|c|}{ Kafr Elhamam } \\
\hline \multirow[t]{2}{*}{ PH } & G & 0.2033 & -0.2203 & 0.0265 & 0.0403 & 0.0217 & -0.0590 & 0.0140 \\
\hline & $\mathbf{P h}$ & -0.0716 & 0.1256 & -0.0107 & -0.0111 & -0.0037 & 0.0062 & -0.0057 \\
\hline \multirow[t]{2}{*}{ NTP } & G & 0.0228 & -1.9644 & 0.8718 & 1.1056 & 0.2876 & 0.2613 & 0.2860 \\
\hline & $\mathbf{P h}$ & -0.0069 & 1.3125 & -0.2461 & -0.2597 & -0.0314 & -0.0370 & -0.1157 \\
\hline \multirow[t]{2}{*}{$\overline{F L}$} & G & 0.0039 & -1.2326 & 1.3893 & 0.2257 & 0.1213 & -0.3302 & -0.0292 \\
\hline & $\mathbf{P h}$ & -0.0016 & 0.6747 & -0.4787 & -0.0790 & -0.0126 & -0.0331 & 0.0063 \\
\hline \multirow[t]{2}{*}{ NSP } & G & 0.0053 & -1.3905 & 0.2008 & 1.5620 & 0.1642 & -0.4469 & 0.1058 \\
\hline & $\mathbf{P h}$ & -0.0018 & 0.7549 & -0.0837 & -0.4516 & -0.0211 & 0.0540 & -0.0378 \\
\hline \multirow[t]{2}{*}{ SL } & G & 0.0054 & -0.6874 & 0.2051 & 0.3121 & 0.8218 & -0.4565 & 0.0045 \\
\hline & $\mathbf{P h}$ & -0.0024 & 0.3699 & -0.0542 & -0.0855 & -0.1115 & 0.0485 & -0.0339 \\
\hline \multirow[t]{2}{*}{ NSS } & G & 0.0077 & 0.3298 & 0.2948 & 0.4486 & 0.2411 & -1.5561 & 0.1553 \\
\hline & $\mathbf{P h}$ & -0.0019 & -0.2037 & 0.0664 & -0.1022 & -0.0227 & 0.2384 & -0.0454 \\
\hline \multirow[t]{2}{*}{$1000 \mathrm{GW}$} & G & 0.0032 & -0.6258 & -0.0459 & 0.1870 & 0.0042 & -0.2735 & 0.8836 \\
\hline & $\mathbf{P h}$ & -0.0012 & 0.4608 & 0.0092 & -0.0519 & -0.0115 & 0.0329 & -0.3295 \\
\hline
\end{tabular}

Abbreviations: PH: Plant height, NTP: No. tillers/plant, FL: Flag Leaf area, NSP: Number of spike/plant, SL: Spike length, NSS: No. spikelets/spike, 1000 GW: weight 1000 grains, Y: Grain yield /ard/fed 
Table.9 The relative importance (RI \%) for six predictor characters on grain yield/ plant at genotypic and phenotypic levels computed from 6 wheat varieties evaluated in two locations

\begin{tabular}{|c|c|c|c|c|c|}
\hline \multirow{2}{*}{ Characters } & \multirow[t]{2}{*}{ Locations } & \multicolumn{2}{|c|}{ AE. } & \multicolumn{2}{|c|}{ KE. } \\
\hline & & Genotypic & Phenotypic & Genotypic & Phenotypic \\
\hline \multicolumn{6}{|c|}{ Direct effect } \\
\hline \multicolumn{2}{|l|}{ Plant height x1 } & 0.0657 & 0.0023 & 0.1351 & 0.1061 \\
\hline \multicolumn{2}{|l|}{ Number of tillers/plant $\mathrm{x} 2$} & 24.0072 & 20.9871 & 12.6185 & 35.6356 \\
\hline \multicolumn{2}{|l|}{ Flag Leaf area $\mathrm{x} 3$} & 1.3380 & 0.0008 & 6.3113 & 4.7396 \\
\hline \multicolumn{2}{|l|}{ Number of spike/plant $x 4$} & 6.2366 & 3.3080 & 7.9779 & 4.2178 \\
\hline \multicolumn{2}{|l|}{ Spike length x5 } & 0.8472 & 3.5088 & 2.2082 & 0.2574 \\
\hline \multicolumn{2}{|l|}{ Number of spikelets/spike x6 } & 14.8217 & 7.3535 & 7.9176 & 1.1761 \\
\hline \multicolumn{2}{|l|}{ Weight of 1000 grains x7 } & 1.6891 & 0.2278 & 2.5532 & 2.2455 \\
\hline \multicolumn{6}{|c|}{ Indirect effects } \\
\hline \multirow{6}{*}{$\begin{array}{l}\text { Plant height }\left(\mathbf{X}_{1}\right) \\
\text { Via }\end{array}$} & $\mathbf{X}_{2}$ & 0.1648 & 0.0201 & 0.2928 & 0.3723 \\
\hline & $\mathbf{X}_{3}$ & 0.0110 & 0.0001 & 0.0352 & 0.0316 \\
\hline & $\mathbf{X}_{4}$ & 0.0424 & 0.0046 & 0.0536 & 0.0329 \\
\hline & $\mathbf{X}_{5}$ & 0.0140 & 0.0062 & 0.0288 & 0.0109 \\
\hline & $\mathbf{X}_{6}$ & 0.0839 & 0.0033 & 0.0784 & 0.0183 \\
\hline & $\mathbf{X} 7$ & 0.0124 & 0.0005 & 0.0186 & 0.0168 \\
\hline \multirow{5}{*}{$\begin{array}{l}\text { Number of tillers/ plant }\left(\mathbf{X}_{2}\right) \\
\text { Via }\end{array}$} & $\mathbf{X}_{3}$ & 3.4634 & 0.0538 & 11.1994 & 13.3608 \\
\hline & $\mathbf{X}_{4}$ & 13.3040 & 8.7746 & 14.2039 & 14.1021 \\
\hline & $\mathbf{X}_{5}$ & 0.7235 & 1.7473 & 3.6945 & 1.7072 \\
\hline & $\mathbf{X}_{6}$ & 12.3692 & 5.4352 & 3.3565 & 2.0097 \\
\hline & $\mathbf{X 7}$ & 3.8850 & 1.3318 & 3.6746 & 6.2814 \\
\hline \multirow{4}{*}{$\begin{array}{l}\text { Spike length }\left(\mathbf{X}_{\mathbf{3}}\right) \\
\text { Via }\end{array}$} & $\mathbf{X}_{4}$ & 0.8907 & 0.0136 & 2.0508 & 1.5638 \\
\hline & $\mathbf{X}_{5}$ & 0.0129 & 0.0101 & 1.1020 & 0.2501 \\
\hline & $\mathbf{X}_{6}$ & 1.7634 & 0.0019 & 3.0001 & 0.6549 \\
\hline & $\mathbf{X} 7$ & 0.2601 & 0.0004 & 0.2652 & 0.1253 \\
\hline \multirow{3}{*}{$\begin{array}{l}\text { Number of spikelets/spike }\left(\mathbf{X}_{4}\right) \\
\text { via }\end{array}$} & $\mathbf{X}_{5}$ & 1.1314 & 1.9944 & 1.6770 & 0.3947 \\
\hline & $\mathbf{X}_{6}$ & 5.2558 & 2.8828 & 4.5656 & 1.0079 \\
\hline & $\mathbf{X 7}$ & 0.9992 & 0.3192 & 1.0805 & 0.7069 \\
\hline \multirow[t]{2}{*}{ Number of grains/spike $\left(X_{5}\right)$ via } & $\mathbf{X}_{6}$ & 2.2397 & 2.7551 & 2.4533 & 0.2239 \\
\hline & $\mathbf{X} 7$ & 0.3304 & 0.2449 & 0.0240 & 0.1566 \\
\hline Number of spikelets/spike (x6) & $\mathbf{X 7}$ & 1.9780 & 0.4838 & 1.5806 & 0.3723 \\
\hline \multicolumn{2}{|l|}{ Total (direct + indirect) } & 97.9405 & 61.4718 & 94.1574 & 91.8541 \\
\hline \multicolumn{2}{|l|}{ Residuals } & 2.0595 & 38.5282 & 5.8426 & 8.1459 \\
\hline \multicolumn{2}{|l|}{ TOTAL } & 100.0000 & 100.0000 & 100.0000 & 100.0000 \\
\hline
\end{tabular}

The bold and underline cells indicate to the highest values.

Abbreviations: PH: Plant height, NTP: No. tillers/plant, FL: Flag Leaf area, NSP: Number of spike/plant, SL: Spike length, NSS: No. spikelets/spike, 1000 GW: weight 1000 grains, Y: Grain yield /ard/fed 


\section{Genotypic and phenotypic correlations}

Phenotypic and genotypic correlation coefficients are presented in Table 7. Grain yield showed positive and significant genotypic correlation with number of tiller per plant and number of spikelets/spike $(\mathrm{r}=0.433$ and 0.407$)$ while phenotypic correlation showed positive and significant for number of tiller /plant $(r=0.365)$ in ItayElbarood and with number of tiller/ plant ( $\mathrm{r}=$ 0.871 and $r=0.616$ ) in Kafr Elhamam location over two years in genotypic and phenotypic, respectively. This may may be attributed to extended period of tillering as the result of conducive field conditions (e.g. lower temperature) during the vegetative phase and delaying tillering end. This idea is supported by Fischer, 1985 who stated that tillering extended until intraplant competition is strong enough to limit the availability of resources for the growth of new tillers.

The direct and indirect effects of six predictor characters on grain yield/plant at genotypic and phenotypic levels computed from 6 wheat varieties evaluated in two locations are presented in Tables 8 and 9.

The results of path coefficient analysis (Table 9) exhibited high positive direct effect of number of tiller/plant on grain yield 24.0072 and 20.9971 in Itay-Elbarood and 12.6185 and 35.6356 in Kafr Elhamam) in both genotypic and phenotypic levels, respectively, followed by number of speckles/ spike on grain yield (14.8217 and 7.3535) for genotypic and phenotypic levels, respectively in Itay-Elbarood and for number of spike /plant (7.9779 and 4.2178) for genotypic and phenotypic levels, respectively in Kafr Elhamam over two seasons. Therefore direct selection through number of tillers/ plant trait will be effective for yield improvement. Similar results direct effect on grain yield was reported by Carcia et al., (1991), Dofing and
Knight (1992), Tammam and El-Syed (2000), Okuyama et al., (2004) and Abd El-Mohsen and Abd El-Shafi (2014).

On the other hand, the indirect effect for number of tillers /plant via number of spike per plant exhibited high positive effect (13.304 and 8.77 for genotypic and phenotypic levels, respectively) in ItayElbarood and also, high positive effect (14.21 and 14.102 for genotypic and phenotypic level, respectively) in Kafr Elhamam (Table 9). Also, indirect effect of number of tillers/plant trait via number of spikelets /spike was positive and high (12.37 and 5.44 for genotypic and phenotypic level, respectively) in Itay-Elbarood while it was positive and high through Flage leaf area (11.20 and 13.36 for genotypic and phenotypic level, respectively) in Kafr Elhamam. These results are similar to those obtained by Abd El-Mohsen et al., (2012) and Abd El-Mohsen and Abd El-Shafi (2014).

It is concluded that Gimaza-7, 10 and Misr1 cultivars were the best yielding among the studied cultivars and the Kafr Elhamam location was the top yielding than the other location (Itay-Elbarood). Genotypic and phenotypic correlation analyses in the two locations reflected a strong positive correlation between grain yield and number of tillers per plant, number of spikelets per spike and No. of spike per plant. The path analyses fortified the outcomes of the correlation and indicated that these three traits had high direct effect on grain yield in both locations. It is worth mentioning that some compensatory tendencies among these contributes were noted. Also the inconsistency in the levels of their estimates in terms of correlations and direct effects coupled with the high residual effects suggests an important role of environmental modification and genetic variability effect on performance of the investigated cultivars from year to year. 
However, it could be concluded that number of tillers per plant, number of spikelets per spike and No. of spike per plant warrant attention of wheat breeder for improving grain yield.

\section{References}

Abd El- Mohsen, A. A.; Abo Hegazy, S. R. and Taha, M. H. 2012. Genotypic and phenotypic interrelationships among yield and yield components in Egyptian bread wheat genotypes. J of Plant Breeding and Crop Sci., 4(1): 9-16.

Abd El-Kreem, T. H. A. and Ahmed, E. G. G. 2013. Evaluation of four new bread wheat (Triticum aestivum L.) cultivars in sandy soils under different irrigation regimes and nitrogen fertilizer rates for yield and its components. Alex. J. Agric. Res., 58 (3): 241-250.

Abd El-Mohsen, A. A. and Abd El-Shafi, M. A. 2014. Regression and path analysis in Egyptian bread wheat. J. of AgriFood and Appl. Sci., Vol. 2(5): 139148.

Ali, Y.; Atta, B. M. Akhter, J.; Monneveux, P. and Lateef, Z. 2008. Genetic variability, association and diversity studies in wheat (Triticum aestivum L.) germplasm. Pak. J. Bot., 40(5): 20872097.

Ashmawy, F.; El-Habal M. S.; Saudy, H. S. and Abbas I. K. 2010. The relative contribution of yield components to grain yield of some wheat cultivars grown under different nitrogen fertilizer levels. Egypt. J. Agric. Res., 88 (1):225239.

Atia, A. A. M. 2007. PATHCA: A BASIC program for estimating phenotypic, genotypic and environmental path coefficient, an application on maize. The 42nd Annual Conf. of Statistics, Computer Sciences and Operation Research, Institute of Statistical Studies and Research. Cairo Univ., 76-87, Egypt, 2-5 December (2007).

Aycicek, M. and Yildirim, T. 2006. Path coefficient analysis of yield and yield components in bread wheat (Triticum aestivum L.) genotypes. Pak. J. Bot., 38(2): 417-424.

Baloch, M. J.; Baloch E.; Jatoi, W. A. and Veesar, N. F. 2013. Correlations and heritability estimates of yield and yield attributing traits in wheat (Triticum aestivum L.). Pak. J. Agri., Eng., Vet. Sci., 29(2): 96-105

Dewey, D. R. and Lu, K. H. 1959. A correlation and path coefficient analysis of components of crested wheat grass seed production. Agron. J. 51: 515-518.

Dofing, S.M. and C.W. Knight. (1992). Alternative model for path analysis of small grain yield. Crop Sci., 32: 487489.

Fischer, R. A. 1985. Number of Kernels in Wheat Crops and the Influence of Solar Radiation and Temperature. J. Agric. Sci. (Cambridge). 108:447-461.

Garcia, M.L.F., F.M. Ramos, M.B. Garcia and T.M.P. Jimenez. (1991). Ontogenetic approach to grain production in spring barley based on path coefficient analysis. Crop Sci., 31: $1179-1185$.

Grafius, J.S. (1959). Heterosis in barley. Agron. J., 51:551-554.

Kashif, M. and Khaliq, I. 2004. Heritability, correlation and path coefficient analysis for some metric traits in wheat. Int. J. Agri. Biol., 6 (1):138-142.

Khan, M. H. and Dar, A. 2010. Correlation and path coefficient analysis of some quantitative traits in wheat. Afr. Crop Sci. J., 18(1): 9-14.

Mehmet, A. and Y. Telat (2006) Path coefficient analysis of yield and yield components in bread wheat (Triticum aestivum L.) Pak. J. Bot., 38(2):417424. 
Okuyama L. A., L. C. Federizzi and J. F. B. Neto. (2004). Correlation and Path Analysis of Wheat and Its Components and Plant Traits in Wheat. Cienc. Rural. Vol.34 No. 6.

Singh P. and S.S. Narayanan (2000): Biometrical techniques in plant breeding. Kalyani Publishers, New Delhi.

Singh R.K. and B.D. Chaudhary (1999):
Biometrical methods in quantitative genetic analysis. Kalyani Publishers, New Delhi

Tamman, A. M., Ali, S. A. and El-Sayed, E. A. M. 2000. Phenotypic, genotypic correlation and path coefficient analysis in some bread wheat crosses. Assiut. J. Agric. Sci., 31(3): 73-85.

Wright, S. (1921): The theory of path coefficient. Genetics, 8: 239-285.

\section{How to cite this article:}

Sahar A. Farag, S.K.A. Ismail and Sawsan A. El-Ssadi. 2018. Estmating Phenotypic and Genotypic Path Coefficient, an Application on Wheat (Triticum aestivum L.) Genotypes. Int.J.Curr.Microbiol.App.Sci. 7(02): 2494-2505. doi: https://doi.org/10.20546/ijcmas.2018.702.304 